\title{
MUESTREO Y FLUCTUACIÓN POBLACIONAL DE MOSCA DEL ESTABLO EN RASTROJO DE PIÑA'
}

Yannery Gómez-Bonilla²

\section{RESUMEN}

Muestreo y fluctuación poblacional de mosca del establo en rastrojo de piña. El objetivo fue determinar la cantidad de la mosca del establo en el rastrojo de piña en derriba en seco. El estudio se realizó en la Finca Babilonia-Siquirres, Costa Rica de agosto a diciembre del año 2012, en una hectárea de rastrojo de piña. El tamaño de la muestra se obtuvo por medio de la Ley de Taylor. Para la captura de adultos, se colocaron cuatro trampas blancas con pegamento al borde de las parcelas de $50 \times 40 \mathrm{~m}$. Para el conteo de larvas se ocuparon las mismas cinco parcelas, se lanzó seis veces al azar un marco de 0,0625 $\mathrm{m}^{2}$, se tomó la planta de piña y el suelo a $20 \mathrm{~cm}$ de profundidad. En el tallo de piña en descomposición y en trampas blancas se encontró una alta población de mosca del establo, doméstica y Euxesta sp. La mosca del establo exhibió un patrón de distribución agregada en el cultivo. Se determinó, que el tamaño de muestra representativa por hectárea para larvas, fue de seis puntos de muestreo y para adultos una trampa. La fluctuación poblacional de la mosca del establo a través del tiempo en rastrojo de piña fue en aumento, después de la quema con fuego la población bajo en un 50 \% y al incorporar el rastrojo al suelo, la población disminuyó en un 80 \%. Se pudo comprobar que hay salida de mosca después de incorporado al suelo el rastrojo.

Palabras clave: Ley de Taylor, Stomoxys calcitrans L., tamaño de muestra, mosca doméstica, Euxesta sp.

\section{INTRODUCCIÓN}

La mosca del establo (Stomoxys calcitrans L.), es una plaga cosmopolita que produce pérdidas directas en la producción de carne y leche como resultado de sus hábitos hematófagos (Mramba et al. 2007). Estos insectos son un problema tanto para animales de vida silvestre, como para animales domésticos y humanos (Zumpt 1973).
Las variaciones de población han sido estudiadas en diferentes países, con el fin de conocer los meses de mayor abundancia (RodríguezBatista et al. 2005, Taylor et al. 2007, Gilles et al. 2007, Keawrayup et al. 2012). Es importante tener conocimiento de la distribución de una plaga porque permite tener un enfoque selectivo

1 Este estudio forma parte de un proyecto de investigación financiado por el INTA y la Fundación para el Fomento y Promoción de la Investigación y Transferencia de Tecnología Agropecuaria (FITTACORI), San José, Costa Rica.

2 Instituto Nacional de Innovación y Transferencia en Tecnología Agropecuaria (INTA). ygomez@inta.go.cr Apartado Postal 10094, San José, Costa Rica. 
y la implementación de medidas de control en el tiempo y espacio, puede ayudar a realizar un manejo integrado plagas (MIP) (Orenstein et al. 2003). En Costa Rica, el primer reporte de mosca del establo se dio por parte de algunas fincas ganaderas debido a la abundancia relativa en ciertos meses del año (Herrero et al. 1989, Herrero et al. 1991).

Esta mosca se alimenta principalmente en las patas, en los flancos de las reses. Los Machos y las hembras chupan sangre dos o tres veces al día, aproximadamente durante cinco minutos y cuando no se están alimentando suelen ubicarse fuera del hospedero, en las paredes $u$ objetos próximos (Chong-Chin et al. 2011).

Asociado al problema de la mosca del establo, se encuentra otra importante plaga como es la mosca doméstica que es una de las plagas más comunes y extendidas en el mundo, es una plaga problemática como vector de más de 100 patógenos serios (virus, bacterias, hongos, protozoos y nematodos) (Davies et al. 2016, Hernández et al. 2012, Zumbado 2006). Las moscas domésticas reproducen fácilmente, una hembra puede poner hasta 500 huevos, y en áreas tropicales esta especie puede darse hasta 20 generaciones / año (Stensmyr 2013 citado por Hamid et al. 2016). Estos insectos son considerados una de las peores plagas domésticas, superando a los mosquitos, hormigas y cucarachas (Zumbado 2006).

Estas dos plagas han venido en aumento en el rastrojo de piña con la introducción de la variedad de piña MD-2. Se intensificó la siembra de este cultivo en Costa Rica, en el año 2014 donde se reportaron 37 659,9 ha sembradas (INEC 2015), de las cuales un $47,5 \%$ se encuentra en la zona Huetar Norte, que es donde más número de brotes de mosca de establo se reporta (SENASA 2012). Después de la segunda cosecha, las plantas de piña se cortan y se incorporan en el suelo en preparación para la replantación.

Lo primero a considerar son los muestreos para poder determinar los principales factores que conllevan a tipificar una muestra para insectos los cuales son: la selección de la unidad de muestreo, la determinación del tamaño de muestra, y el programa de muestreo (Veccol et al. 2015). Se debe conocer el patrón de dispersión espacial de la plaga. La Ley de Taylor permite definir el tipo de dispersión de la plaga, lo cual sería un gran aporte al poder contar con el tamaño de la muestra $\mathbf{n}$ expresada en términos de sus coeficientes (Navarro-Flores 1992, Giraldo-Mendoza et al. 2002, Kalsi et al. 2014, Arbab y Mirphakhar 2016). Se han realizado investigaciones con otros insectos como la mosca blanca, trips, algunos dípteros, coleópteros y otros; donde se ha establecido que la Ley de Taylor es robusta para determinar la distribución (Socías et al. 2014, Stubbins et al. 2014, Zhang et al. 2015, Veccol et al. 2015, Zheng-Young et al. 2015, Arbab y Mirphakhar 2016).

El objetivo fue determinar la cantidad de la mosca del establo en el rastrojo de piña en derriba en seco.

\section{MATERIALES Y MÉTODOS}

La presente investigación se llevó a cabo en una hectárea de la Finca Babilonia de PINDECO, en el cantón de Siquirres, provincia de Limón, Costa Rica. La zona se clasifica según las zonas de vida de Holdridge (Bolaños et al. 1998) como húmedo tropical. El periodo del estudio comprendió los meses de agosto a principios de diciembre del año 2012. En la zona se registró un rango de temperatura de 25 a $29^{\circ} \mathrm{C}$, una humedad relativa de 80 a 95 \% y la precipitación varió desde
150 a 780 mm (Estación climática de PINDECO: Davis Vantage Pro, Vernon Hills, IL).

\section{Eliminación de la plantación (derriba)}

Posterior a la cosecha del fruto, las plantas se sometieron a derriba, la cual consistió en la aplicación del herbicida paraquat a 2 PF/ha mediante una aplicación con un boom de 40 boquillas 
acoplada a un tractor. El herbicida deseca la planta hasta secar las hojas, luego de casi 30 días se quemaron con fuego, se trituraron, y se incorporaron al suelo por medio de una pasada de rastra de discos. En el muestreo se incluyeron todas estas etapas debido a las posibles variaciones de las poblaciones del insecto.

Se realizó una prueba de $t$ de Student utilizando el programa estadístico InfoStat (Di Rienzo et al. 2008), para comparar las poblaciones de mosca del establo antes y después del fuego, así como de antes y después de incorporar el rastrojo al suelo y así cuantificar el efecto de esa práctica.

\section{Muestreo de larvas}

Para el conteo de larvas se tomaron muestras en una hectárea de terreno previo al iniciar la derriba del cultivo. El área se dividió en cinco parcelas del mismo tamaño con un área de $2000 \mathrm{~m}^{2}$. Dentro de cada parcela se caminaba en un transepto en diagonal, tratando de cubrir el área, se utilizó como una unidad de muestreo un cuadro de metal de 0,25 x 0,25 m $\left(0,0625 \mathrm{~m}^{2}\right)$, el cual fue lanzado al azar, cinco veces en cada parcela, para un total de 25 unidades muestrales por hectárea. Este procedimiento se realizó cada ocho días durante 10 semanas (hasta la incorporación del material al suelo). De cada unidad de muestreo se tomaron $20 \mathrm{~cm}$ de suelo, aproximadamente un kilogramo y la planta completa de piña, la cual se colocó en una bolsa plástica y se llevó al laboratorio para su análisis. En el laboratorio con una lupa de 40x y un estereoscopio (Nikon, Mod: C-Lex) se revisaron las muestras, se separaron por morfología y contaron las larvas y pupas de las especies más abundantes y se llevaron a identificar al Instituto Nacional de Biodiversidad (INBio), para determinar las especies presentes en los rastrojos.

\section{Muestreo de adultos}

Para el muestreo de adultos de mosca del establo se colocaron 4 trampas/parcela de bolsas plásticas $(0,8 \times 0,60 \mathrm{~m})$ color blanco con pegamento (Zapicol mezclado con gasolina comercial); en las mismas cinco parcelas del muestreo de larvas, en el borde de cada parcela se colocó una trampa blanca en cada punto cardinal, para un total de 20 trampas/ha. Cada bolsa estaba sujetada por dos palos de madera a $20 \mathrm{~cm}$ del suelo (Figura 1). Al igual que con las larvas este procedimiento se realizó cada ocho días durante 10 semanas (después de la incorporación del material al suelo, se dejaron las trampas una semana más y únicamente se contó adultos de la mosca del establo). Cada semana se realizó el conteo de los insectos pegados y se identificó las especies más abundantes en ambas caras, para conocer su fluctuación poblacional a través del tiempo en el estudio. Una vez contados los insectos de la bolsa, inmediatamente se reemplazaba por una nueva.
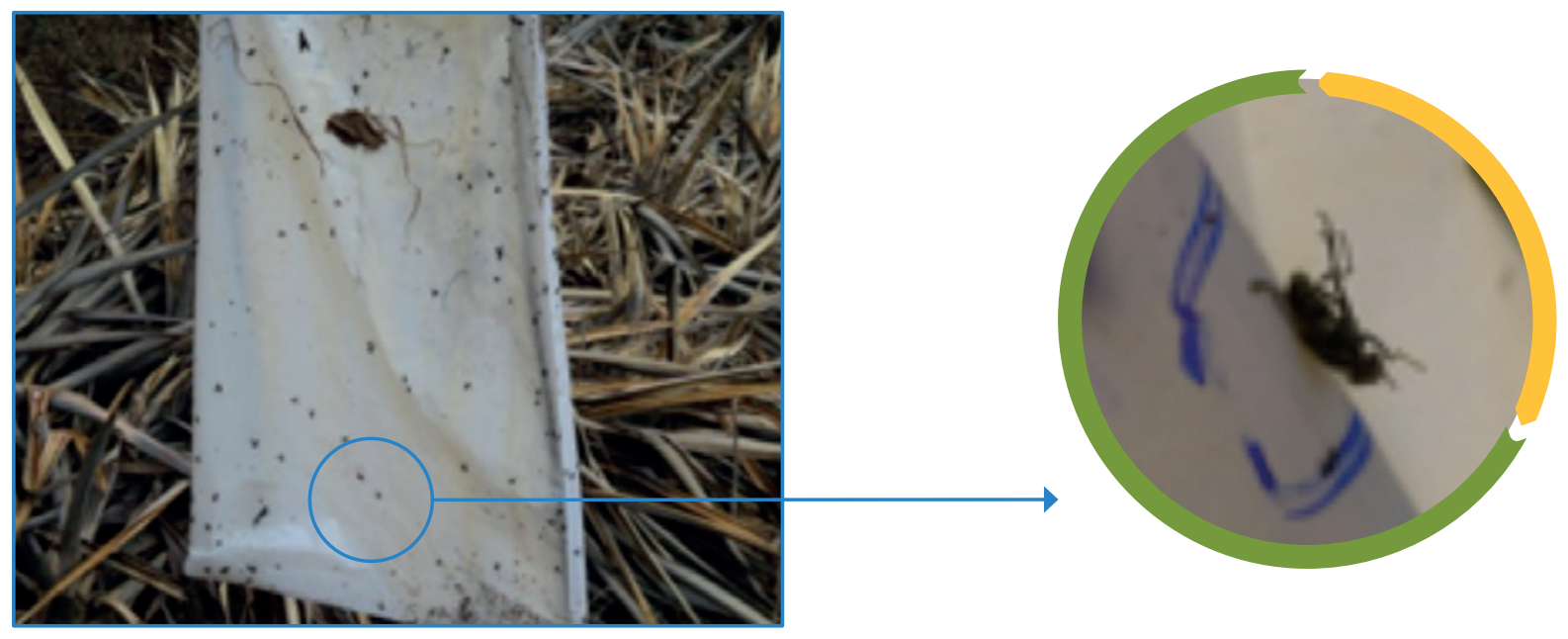

Figura 1. Adulto de mosca del establo en trampa blanca con pegamento en rastrojo de piña. Finca Babilonia Siquirres, Costa Rica, 2012. 
Para poder comprobar la salida de las moscas en el rastrojo de piña, se colocaron tres tipos de trampas en un metro cuadrado, en las semanas 8 y 9 , en cada una de las parcelas anteriormente descritas (Figura 2). La trampa A utiliza botellas plásticas transparentes de 2-3 litros. La mosca es atraída por la luz y queda capturada en la botella, esta trampa tiene la ventaja que captura la mosca viva. (Diseño original del PINDECO). La trampa B es una trampa con una bolsa blanca pintada con pegamento, se utilizó de dos formas, en forma vertical, que es la más utilizada y $C$ forma horizontal a $20 \mathrm{~cm}$ sobre el suelo (Diseño original de Ing. Dennis Alpízar Monge del INTA). Todas las trampas fueron cubiertas por un sarán, para que únicamente las moscas del suelo tengan la posibilidad de ingresar a la trampa.

Se realizó ANDEVA con el programa InfoStat, para comparar las poblaciones a través del tiempo de las moscas capturadas y los tipos de trampas.
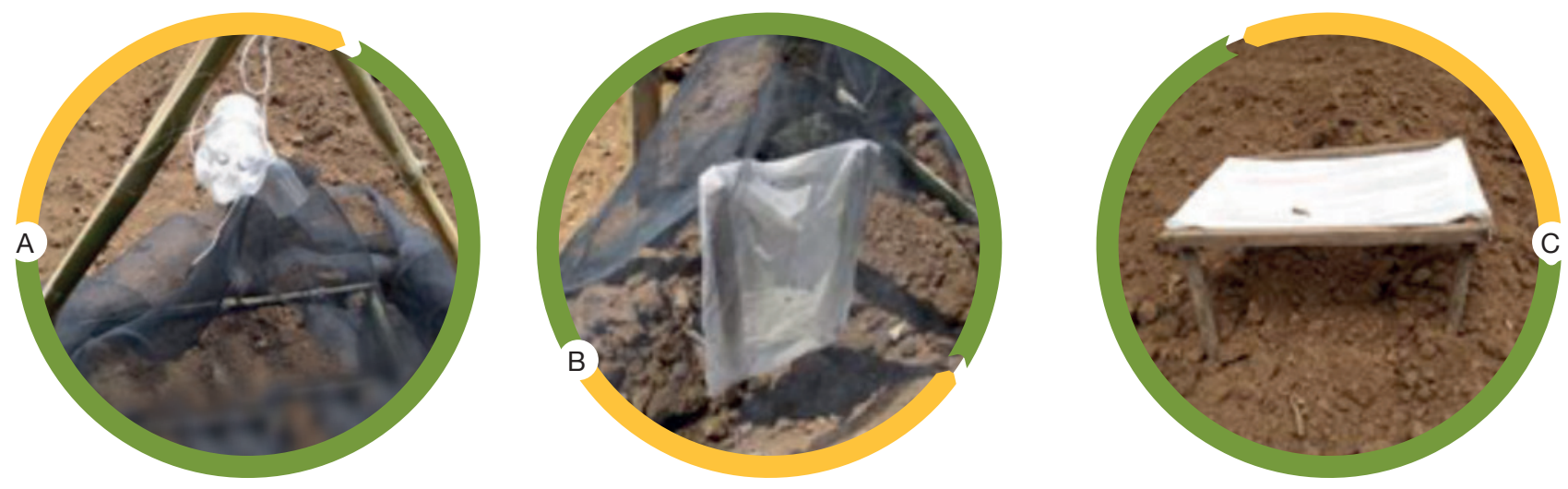

Figura 2. Tipos de trampas utilizadas, para captura de adultos que salen de los rastrojos de piña. Finca Babilonia Siquirres, Costa Rica, 2012.

\section{Tamaño de muestra, larvas y adultos}

Para determinar el tamaño de la muestra para larvas y adultos de la mosca del establo, se siguió la Ley de Taylor (Taylor 1984), la cual explica la relación entre la media y la variancia, $S^{2}=a m^{b}$. Los coeficientes de Taylor pueden calcularse de acuerdo con la siguiente ecuación: $\log \boldsymbol{S}^{2}=\log \boldsymbol{a}+\boldsymbol{b} \log \boldsymbol{m}$ (Duque- Echeverri 1996, Giraldo- Mendoza et al. 2002, Barrera et al. 2006). Esta es una simple regresión en donde se contrastan las variancias de diversas muestras contra sus respectivas medias, todo transformado en logaritmos. En la práctica, b es considerado como una medida específica de la especie de dispersión de los recuentos en el muestreo, si b>1 (los patrones espaciales indica que la población se encuentra agregado), si $b=1$ (se encuentra al azar) y si $b<1$ (se encuentra en forma regular).
Finalmente, a es un factor de muestreo que tiene menos interés para definir el patrón de agregación (Taylor 1984, Kuno 1991).

Una vez obtenidos los valores de a y $b$ de la anterior ecuación, se utilizó la fórmula que se describe a continuación para obtener el tamaño de muestra.

$$
\mathbf{n}=\frac{\mathbf{a x ^ { - ( b - 1 ) }}}{\mathbf{c}}
$$

Donde: n: tamaño de muestra, a: parámetro que representa Intercepto de la ecuación, b: parámetro que representa pendiente, $x=$ promedio de las muestras realizadas, c: precisión, escogida de $90 \%$. 


\section{Análisis de datos}

Se realizó la prueba de $t$ de Student para comparar las poblaciones de mosca del establo antes y después del fuego y de incorporar el rastrojo al suelo y así cuantificar el efecto de esas prácticas.
En el caso de los muestreos semanales se realizó ANDEVA con un modelo lineal generalizado con repeticiones en el tiempo, para los conteos de adultos de las diferentes especies para determinar el efecto de las prácticas de manejo del cultivo sobre las poblaciones, con separación de medias utilizando prueba de Tukey, en el programa InfoStat.

\section{RESULTADOS Y DISCUSIÓN}

\section{Identificación de especies de moscas encontradas en el rastrojo de piña}

Las plantas de piña fueron revisadas exhaustivamente. No se encontraron indicios de ningún tipo de larva en plantas de piña verde, las primeras larvas se encontraron a partir del inicio de la descomposición de las plantas de piña, lo cual ocurrió luego de dos semanas de la derriba.

En el rastrojo de piña, se identificaron dos especies de larvas en mayor abundancia: la mosca del establo, donde las larvas se pueden identificar por el tipo de espiráculos bien separados y en forma triangular (Figura 3) y Euxesta sp. (Figura 4), cuyos espiráculos se encuentran expuestos. En el caso de los adultos, en las trampas con pegamento se encontraron en gran abundancia tres especies, las dos citadas anteriormente y la mosca doméstica (Musca domestica) donde las larvas tienen sus espiráculos más juntos y en forma arriñonada (Figura 5). Todas estas moscas son atraídas por material en descomposición (Birkemoe y Sverdrup-Thygeson 2011).

En las mismas tres figuras se muestran las pupas de cada especie, que tienen las mismas características de los espiráculos que las larvas. Los adultos, de la mosca del establo se caracterizan por poseer una probóscide en forma de aguja, la doméstica tiene una probóscide más gruesa y en forma de esponja y Euxesta sp, se caracteriza porque posee alas con manchas oscuras en líneas horizontales. En las muestras de suelo no se encontró ningún tipo de insecto.
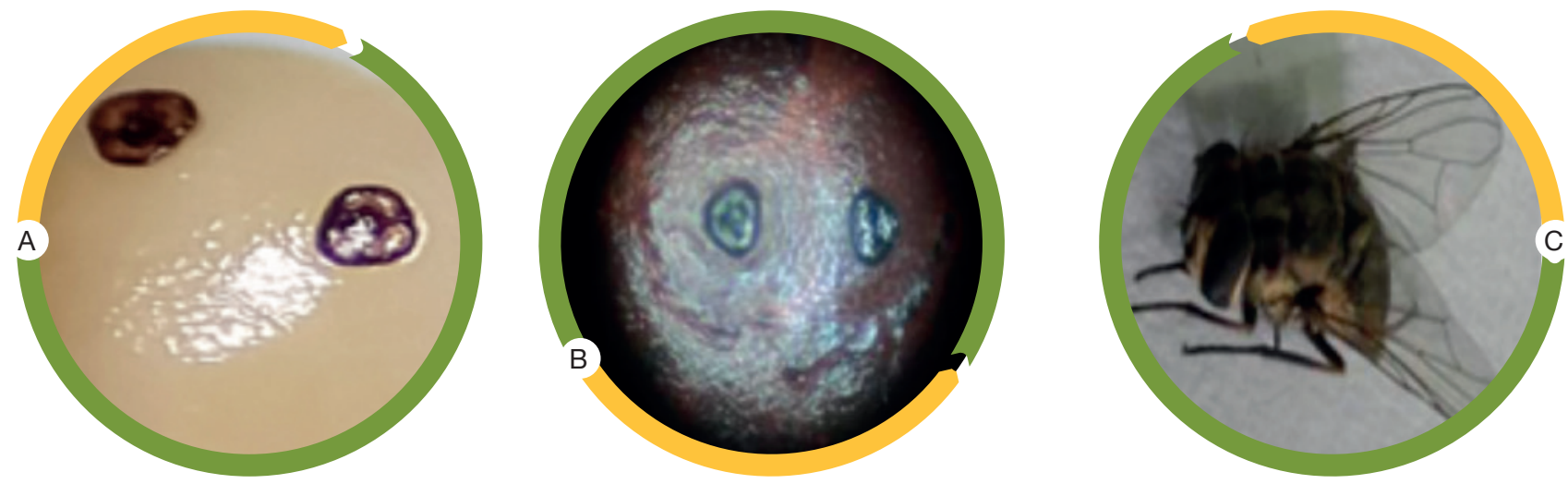

Figura 3. Larva (A), pupa (B), adulto de mosca del establo (C). Finca Babilonia Siquirres, Costa Rica, 2012. 

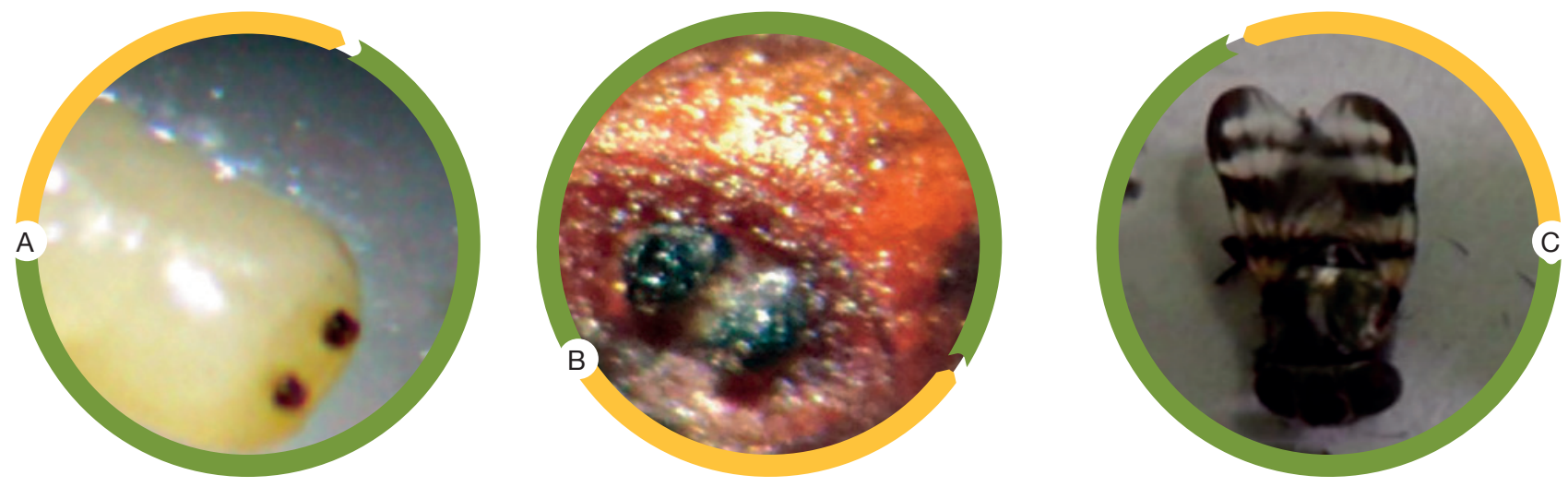

Figura 4. Larva (A), pupa (B), adulto de Euxesta sp. (C). Finca Babilonia, Siquirres, Costa Rica, 2012.
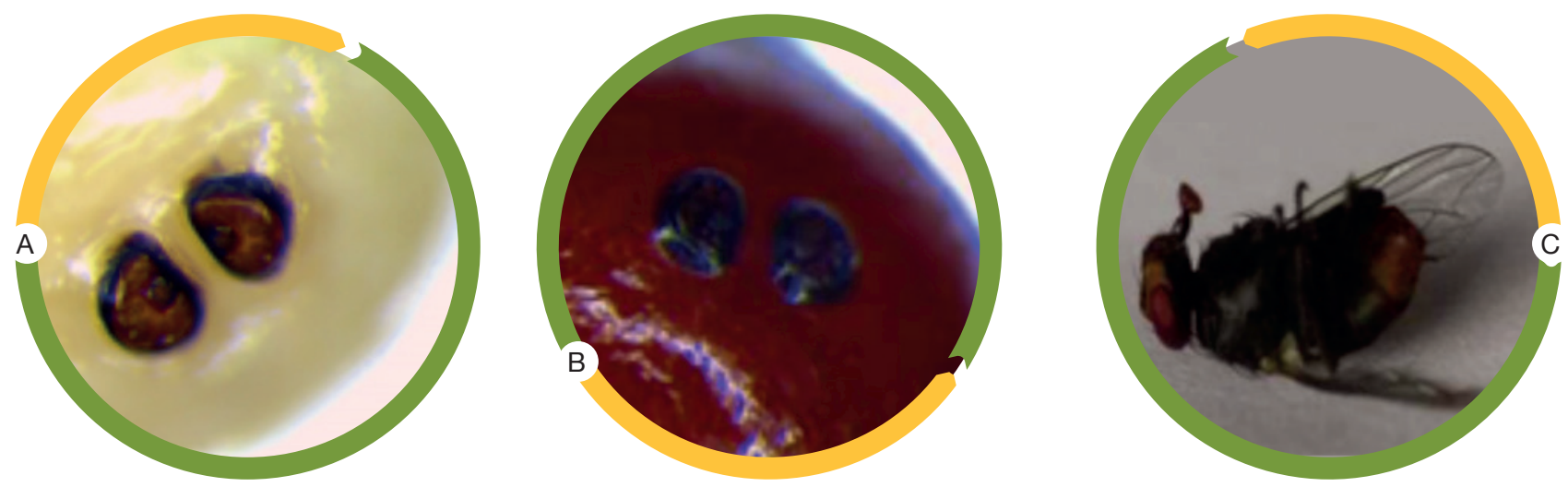

Figura 5. Larva (A), pupa (B), adulto de mosca doméstica (C). Finca Babilonia, Siquirres, Costa Rica, 2012.

\section{Tamaño de muestras y distribución espacial de larvas y adultos de la mosca del establo}

LARVAS: Los resultados que se muestran en el Cuadro 1, fueron para calcular el tamaño de muestra para larvas de la mosca del establo, se obtuvo una $R^{2}$ de 0,7 y la $p=0,0789$, para 43 puntos de muestreo en una hectárea de rastrojo. Esto representa un número de muestras bastante alto, lo que conllevaría mucho trabajo y tiempo para los que van a tomar la muestra y revisarla; también es difícil de manejar debido a la cantidad de larvas de diferentes especies que se encontraron en cada tallo. Las larvas tienen tamaños de $(2-6 \mathrm{~mm})$, muy parecidos y difíciles de diferenciar a simple vista. Este material vegetal que se encuentra en proceso de descomposición desprende mal olor, lo que dificulta trabajar por largos periodos de tiempo. Por esas razones se consideró el ajuste de tamaño de muestra, que sugiere Taylor, mediante una transformación lineal, de la media y la varianza. Al transformar los datos, mejoró la $R^{2}=0,89$ y la probabilidad fue significativa $p=0,004$. El tamaño de la muestra obtenido fue de seis puntos de muestreo/hectárea (Cuadro 1), este tamaño de muestra debería responder a la disponibilidad de tiempo y recurso. El procedimiento anterior se describe ampliamente en los trabajos de (Kuno 1991 y Navarro 1992).

Por medio de la relación media-varianza descrita en la Ley de Taylor se confirma que la distribución de la larva y adultos de la mosca del establo se da en forma agregada (Cuadro 1) identificando como los sitios de agregación únicamente a las plantas de piña en descomposición. Estos residuos vegetales, combinado con el clima 
húmedo y la alta precipitación, son un excelente substrato para el desarrollo de las larvas de estas especies. La agregación se ha señalado como un patrón común presente en muchas especies de insectos (Faleiro et al. 2002, Salama y AbdeElgawad 2010, Arbab y Mirphakhar 2016). Moradi-Vajargah et al. (2011) observaron un patrón de distribución de agregados para todas las etapas de la vida del gorgojo de la alfalfa Hypera postica Gyllenhal (Coleoptera: Curculionidae) excepto pupas, que exhibió un patrón aleatorio por aumento de la densidad.

ADULTOS: En el caso del monitoreo de adultos de la mosca del establo, se utilizó la misma fórmula, y al igual que con las larvas al transformar los datos, mejora la significancia y la $\mathrm{R}^{2}$. Se determinó que al colocar una trampa/ha, se obtiene información confiable (Cuadro 1). Los tamaños de muestras óptimos van a depender también del nivel de precisión deseado y son una herramienta para la toma de decisiones en la investigación (Arbab y Mirphakhar 2016).

En el monitoreo de adultos con las trampas blancas, se encontró Euxesta sp, moscas domésticas y del establo, estas dos últimas son plagas importantes en las explotaciones ganaderas y a menudo dependiendo del tipo de trampa y del tiempo de exposición se da mayor abundancia de una especie que otra (Birkemoe y SverdrupThygeson 2011). En el caso de mosca del establo los adultos tienen la capacidad de desplazarse a distancias considerablemente largas para ovipositar (Taylor et al. 2010).

Cuadro 1. Patrón espacial y tamaño de muestra usando la Ley de Taylor con datos reales y transformados (trans) para la mosca del establo. Finca Babilonia, Siquirres, Limón, Costa Rica. 2012.

\begin{tabular}{lc|c|c|c|c|c|c|}
\multicolumn{1}{c|}{ Estado } & $\mathbf{n}$ & $\mathbf{A}$ & $\mathbf{b}$ & $\mathbf{R}^{2}$ & $\mathbf{P}$ & Distribución & $\begin{array}{c}\text { Tamaño de } \\
\text { muestra }\end{array}$ \\
\hline Larvas & 150 & 1,55 & 2,59 & 0,7 & 0,0789 & Agregado & 43 \\
Larvas trans & 150 & 0,27 & 0,46 & 0,89 & 0,0045 & Regular & 6 \\
\hline Adultos & 160 & 1,20 & 2,06 & 0,90 & 0,0003 & Agregado & 339 \\
\hline Adultos trans & 160 & 1,12 & 2,9 & 0,93 & 0,0001 & Agregado & 1 \\
\hline
\end{tabular}

$\mathrm{P}<0,05$.

\section{Fluctuación poblacional de moscas encontradas en rastrojo de piña}

En la (Figura 6) se presenta la fluctuación poblacional promedio de adultos de las especies de moscas que se encontraron en el rastrojo de piña en las diferentes fases de la derriba, se dieron diferencias significas en las especies de moscas encontradas $p=0,0067$, y diferencias altamente significativas en las semanas de evaluación $\mathrm{p}=0,0000$. Se encontró mosca del establo, Euxesta sp. y mosca doméstica. En las semanas de evaluación previas cuando el cultivo estaba en verde, no hubo presencia de ninguna de las moscas.

Después de la aplicación del herbicida las hojas de la planta de piña se secaron y se inició la descomposición (semana 2 en adelante), atrayendo las moscas. Es común encontrar a la mosca del establo y doméstica en poblaciones mixtas en materiales en descomposición (Birkemoe y
Sverdrup-Thygeson 2011), la mosca doméstica pueden ser atraída por olores como el complejo de melazas como el que produce el melón (Hung et al. 2015), porque tienen un sistema olfativo bien desarrollado y altamente sofisticado para discriminar las señales volátiles que se liberan donde los sustratos pueden servir para la oviposición y fuentes de alimento (Mustafa et al. 2016), este autor cita a (Hogsette 1996) para indicar que las malas condiciones higiénicas, con un ambiente caliente y húmedo son ideales para la cría de mosca doméstica.

En el muestreo de la semana tres las poblaciones de mosca doméstica y Euxesta no presentaron diferencias significativas entre ellas, pero fueron diferentes a las poblaciones de mosca del establo, donde el número de individuos es mayor, posiblemente se esté iniciando la entrada hacia la plantación para sus oviposiciones. 
En las semanas (4, 5, 6 y 7, Figura 6), la población de mosca del establo y doméstica aumentó significativamente con respecto a las poblaciones de Euxesta sp., los valores promedio del conteo de adultos de la mosca del establo fue de $(38,35,33$ y 30$)$ respectivamente y de mosca doméstica $(35,39,31$ y 34$)$, sin presentar diferencias significativas entre sí, mientras que ambas poblaciones fueron diferentes a las poblaciones de Euxesta sp. (13, 16, 15 y 18). Varios estudios han demostrado que la variación poblacional de la mosca dependerá de los factores ambientales y el tipo de cubierta vegetal, entre otros factores (Rasmussew y Campbell 1981, Mavoungou et. al. 2008, Mavoungou et al. 2013).

Debido a la gran cantidad de moscas (Figura 6 y 7), se procedió a quemar con fuego el área de estudio (semana 7), para bajar la población de moscas, ocurriendo una disminución altamente significativa entre las poblaciones de mosca del establo $(p=0,0080)$, antes de que se realizara el fuego el promedio de mosca del establo fue de 27,8 adultos y se redujo a 14,7 con ésta práctica (semana 8), la disminución de un $50 \%$ es producto de que el fuego elimina material residual y mueren larvas y adultos de todas las especies. Sin embargo, el tronco de piña no se quema en su totalidad y continúa su descomposición, por lo que sigue siendo un rastrojo atractivo para las moscas. Posteriormente se dio la trituración de los tallos de piña e inmediatamente la incorporación al suelo, esta última práctica ayudo a bajar las poblaciones de mosca (semana 10), se dio una disminución altamente significativa entre las poblaciones de la mosca del establo $(p=0,0001)$. Antes de incorporar el material vegetal el promedio de mosca fue de 8,3 adultos y una semana después fue de 1,71 adultos, lo que ayudó a bajar la población de mosca del establo en más de un $80 \%$.

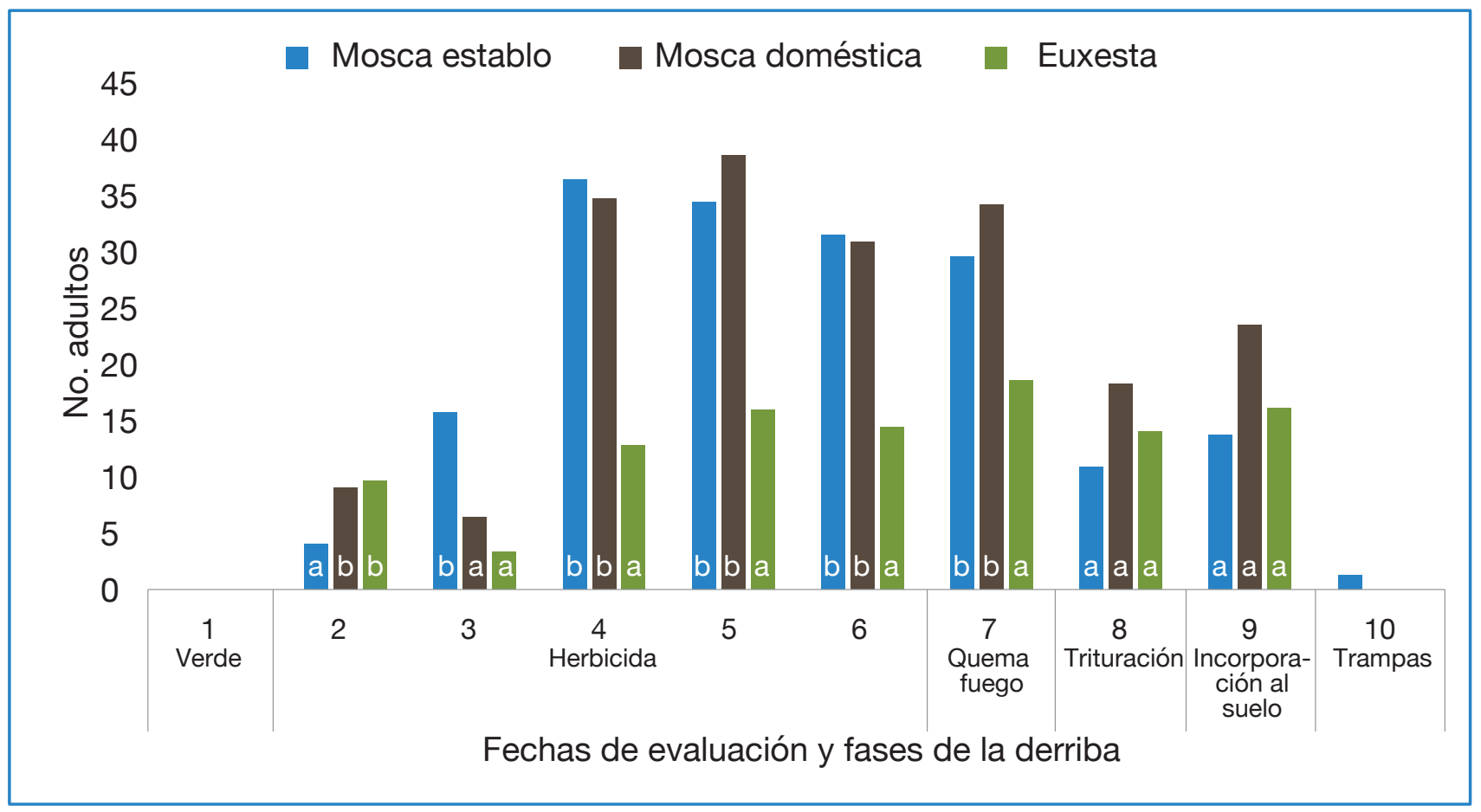

Figura 6. Fluctuación poblacional promedio de adultos de la mosca del establo, doméstica y Euxesta en diferentes fases de la derriba en seco con su respectiva prueba de Tukey. Finca Babilonia, Siquirres, Limón, Costa Rica. 2012. 


\section{Salida de moscas del suelo con el rastrojo incorporado}

Con los tres tipos de trampa, se pudo comprobar la salida de moscas del rastrojo de piña, aunque no se dio diferencias significativas entre las trampas $p=0,1376$, ni entre las especies de moscas ( $p=0,0067)$. En el Cuadro 2, se muestra el número de moscas total capturada de cada una de las especies en las dos fechas de evaluación. Este experimento permitió demostrar que las moscas que posiblemente estén más superficiales, logran salir del rastrojo después de incorporado al suelo. En el material de piña triturado y enterrado es posible que sobrevivan algunos huevos, larvas y pupas, las cuales siguen con su ciclo de vida y logren salir algunos adultos entre la tierra, como se ve en las Figura 6 y 7 . Una semana después de enterrado el rastrojo se capturaron adultos de mosca del establo, lo que indica que es necesario dar seguimiento a la salida de mosca del establo después de incorporado el rastrojo.
Cuadro 2. Salida de moscas después de enterrado el rastrojo de piña con las diferentes trampas de estudio. Finca Babilonia, Siquirres, Limón, Costa Rica. 2012.

\begin{tabular}{l|cc|c|}
\multirow{2}{*}{ Tipo de mosca } & \multicolumn{3}{|c}{ Tipo de trampa } \\
\cline { 2 - 4 } & Vertical & Horizontal & Botella \\
\hline Mosca establo & 19 & 32 & 6 \\
\hline Mosca doméstica & 15 & 22 & 23 \\
\hline Euxesta sp & 15 & 27 & 4 \\
\hline
\end{tabular}

Se observa en la Figura 7 que los rangos de poblaciones de todas las especies son cuantiosos, con la mosca del establo se obtuvo un rango de 100 a 750 adultos, esta cantidad de mosca en el ganado se vería seriamente afectado, ya que la mosca del establo necesita tomar sangre al menos una vez al día (Zumbado 2006), y por eso son consideradas una de las plagas más perjudiciales del ganado en el mundo (Taylor et al. 2012). Pero además se suma una de las plagas más importantes como lo es la mosca doméstica (con una captura de 120-800), que perjudica muchas de las principales industrias, incluidos unidades de procesamiento, ganado y granjas avícolas (Malik et al. 2007).

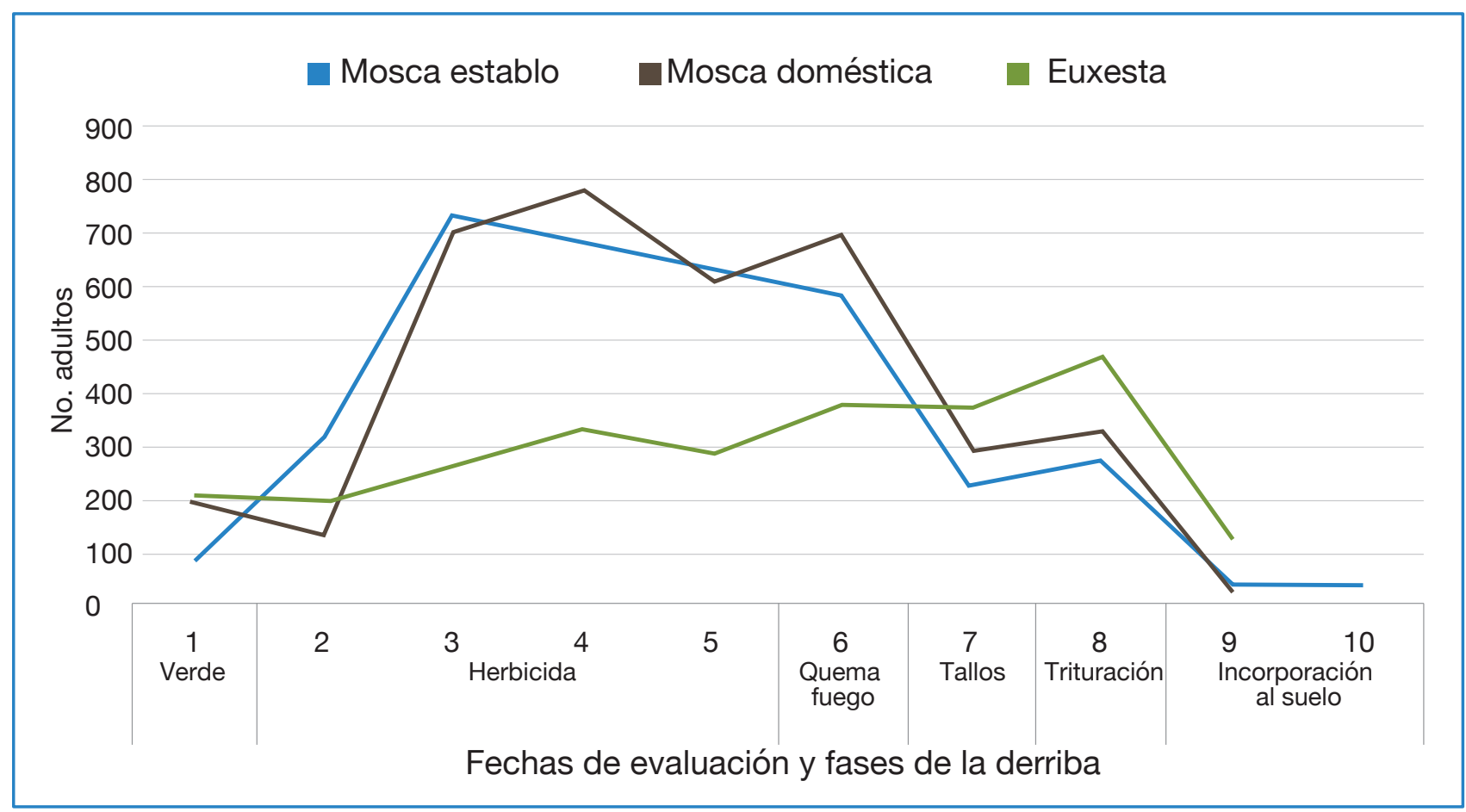

Figura 7. Fluctuación poblacional total de captura de adultos de la mosca del establo, doméstica y Euxesta en rastrojo de piña, en 20 trampas/ha. Finca Babilonia, Siquirres, Limón, Costa Rica. 2012. 


\section{AGRADECIMIENTO}

A PINDECO - Compañía del Monte y su personal técnico.

A Fittacori, por el aporte económico.

A la Ing. Beatriz Sandoval Carbajal, MSc. por sus aportes en el área de estadística.

\section{LITERATURA CITADA}

Arbab, A; Mirphakhar, F. 2016. Spatial distribution pattern and sequential sampling plans for Bactrocera oleae (Gmelin) (Dip:Tephritidae) in olive orchards. J. Entomol. \& Acarolog. 48(1):23-28.

Barrera, JF; Valle, J; Herrera, J; García, H; Villalobos, J; Gómez, J. 2006. "Potencial de la Ley de Taylor para transformar datos de trampeo de broca del café, Hypothenemus hampei." Entomol. Mexicana 5(2):647-653.

Birkemoe, T; Sverdrup-Thygeson, A. 2011. Stable fly (Stomoxys calcitrans) and house fly (Musca domestica) densities: a comparison of three monitoring methods on pig farms. J. Pest Science. 84(3):273-280. DOI: 10.1007/ s10340-011-0352-7.

Bolaños, M; Watson, V; Tosi, J. 1999. Mapa Ecológico de Costa Rica (Zona de Vida). Según el sistema de clasificación de zonas de vida del mundo de L.R.

Holdrigge. Centro Científico Tropical. Sistema de Información Geográfica (en línea). San José Costa Rica. Escala 1:400 000. Color. Consultado Oct. 2015. Disponible en http://www.mediafire.com/ view/?jr1lmseyOuu288z

Chong-Chin, H; Nazni-Wasi, A; Chew-Wai, K; Hiromu, K; John, J; Heah-Sock, K; Baharudin, O. 2011. A Study of Cow Dung Diptera in Sentul Timur, Kuala Lumpur, Malaysia. J. Trop. Med. Parasitol. 33:53-61.

Davies, MP; Anderson, M; Hilton, AC. 2016. The housefly Musca domestica as a mechanical vector of Clostridium difficile. Journal of Hospital Infection. 94(3):263-267. DOI: 10.1016/j. jhin.2016.08.023.

Di Rienzo, JA; Casanoves, F; Balzarini, MG; Gonzalez, L; Tablada, M; Robledo, CW. 2008. InfoStat, versión 2008, Grupo InfoStat, FCA, Universidad Nacional de Córdoba, Argentina.
Duque-Echeverri, M. 1996. Patrones de disposición espacial y su importancia en la definición de un plan de muestreo en MIP. Colombia. Centro Internacional de Agricultura Tropical (CIAT).14 p.

Faleiro, JR; Ashok Kumar, J; Rangnekar, PA. 2002. Spatial distribution of red palm weevil Rhyncophorus ferrugineus Oliv. (Coleoptera: Curculionidae) in coconut plantations. Crop Prot. 21(2):171-176.

Gilles, J; David, JF; Duvallet, G; De la Rocque, S; Tillard, E. 2007. Efficiency of traps for Stomoxys calcitrans and Stomoxys niger niger on Reunion Island. Medical and Veterinary Entomology. 21(1): 65-69. DOI: 10.1111/j.1365-2915.2006.00658.x

Giraldo-Mendoza, A;Véliz-Rosas, C; ArellanoCruz, G; Sánchez-Infantas, E. 2002. El uso de la ley de Taylor en el establecimiento de patrones de variación espacio-temporal en poblaciones animales:dos ejemplos de aplicación. Ecol. Apl. 1(1):71-74.

Hamid, MS; Daud, A; Mohamed, SB; Mohamad, NM; Rashid, MA, 2016. A comparative study on different baits used to attract housefly in Malaysia. International Journal on Advanced Sciencie Engineering Information Technology. 6(5): 588-593.

Herrero, MV; Montes Pico, L; Sanabria, C. 1989. Preliminary studies on the stable fly, Stomoxys calcitrans (Diptera: Muscidae), in the South Pacific region of Costa Rica. Rev. Cienc. Vet. 11(2-3):11-14.

Herrero, MV; Montes Pico, L; Hernández, R. 1991. Relative abundance of Stomoxys calcitrans (L.) (Diptera: Muscidae) in six localities on the southern Pacific coast of Costa Rica. Rev. Biol. Trop. 39(2):309-310. 
Hernández-Escareno, JJ; Renteria-Solis, ZM; Flores-Landaverde, LE; Hernández-Vidal, G; Espinoza-Mata, A. Zarate-Ramos, JJ. 2012. Presence of Enterobacteriaceae, Listeria spp., Vibrio spp and Staphylococcus spp in House fly (Musca domestica L.), Collected and Macerated from Different Sites in Contact with a few Animals Species. Revista Centifica Facultad de Ciencias Veterinarias. 22(2):128-134.

Hogsette, JA. 1996. Development of house flies (Diptera: Muscidae) in sand containing varying amounts of manure solids and moisture. J Econ Entomol. 89(4):940-945

Hung, K; Michailides, TJ; Millar, JG; Wayadande, A; Gerry, A. 2015 House Fly (Musca domestica L.) Attraction to Insect Honeydew. PLOS ONE. 10(5):1-14. DOI:10.1371/journal.pone.0124746.

INEC (Instituto Nacional de Estadística y Censos). 2015. VI Censo Nacional Agropecuario: Resultados Generales. San José, Costa Rica. 147 p.

Kalsi, M; Seal, DR; Nuessly, GS; Capinera, JL; Martin, CG. 2014. Distribution of Zelus longipes (Hemiptera: Reduviidae) in South Florida Corn Fields and Its Functional Response to CornInfesting Picture- Winged Flies (Diptera: Ulidiidae). Environ. Entomol. 43(5):1223-1234.

Keawrayup, S; Duvallet, G; Sukonthabhirom, S; Chareonviriyaphap, T. 2012. Diversity of Stomoxys spp. (Diptera: Muscidae) and diurnal variantions of activity of Stomoxys indicus and S. calcitrans in a farm, in wang nan Khiao District, Nakhon Ratchasima Province, Thailand. Parasite. 19(3): 259-265.

Kuno, E. 1991. Sampling and analysis of insect populations. Annu. Rev. Entomol. 36:285-304.

Malik, A; Singh, N; Satya, S. 2007. House fly (Musca domestica): a review of control strategies for a challenging pest. J. Environ Sci. Health, Part B. $42(4): 453-469$

Mavoungou, JF; Jay-Robert, P; Gilles, J; AtsameEdda, A; Duvallet, G. 2008. Écologie des Stomoxes (Diptera: Muscidae) au Gabon.I- Premier Inventaire dans differentes zones. Ecologiques. Parasite. 15(4):27-34.
Mavoungou, JF; Picard, N; Kohagne, LT; Batch, MT; Chi, AT; Gilles, J; Duval, G. 2013. Spatio-temporal variation of biting flies, Stomoxys spp. (Diptera: Muscidae), along a man-made disturbance gradient, from primary forest to the city of Makokou (North-East, Gabon). Med.Vet. Entomol. 27(3):339 345. doi: 10.1111/j.1365-2915.2012.01064.x

Moradi-Vajargah, M; Golizadeh, A; RafieeDastjerdi, H; Zalucki, P; Hassanpour, M; Naseri, B. 2011. Population density and spatial distribution pattern of Hypera postica (Coleoptera: Curculionidae) in Ardabil, Iran. Not. Bot. Horti Agrobot. 39(2):42-48

Mramba, F; Broce, AB; Zurek, L. 2007. Vector competence of stable flies, Stomoxys calcitrans L. (Diptera: Muscidae), for Enterobacter sakazakii. J. Vector Ecol. 32(1):134-139.

Mustafa S, R; Azhar, F; Shad, SA; Walker, W; Azeem, M; Binyameen, M. 2016. Effects of different animal manures on attraction and reproductive behaviors of common house fly, Musca domestica L. Parasitol Res. 115(9):3585-3598 DOI 10.1007/s00436-016-5124-0

Navarro-Flores, JR. 1992. El muestreo en la investigación con enfermedades y plagas de los cultivos. El diseño experimenal de las plagas. Editado por CIPROC, Universidad de Costa Rica. $421 \mathrm{p}$.

Orenstein, S; Zahavi, T; Nestel, D; Sharon, R; Barkalifa, M; Weintraub, PG. 2003. Spatial dispersion patterns of potential leafhopper and planthopper (Homoptera) vectors of phytoplasma in wine vineyards. Ann. Appl. Biol 142(3):341-348.

Rasmussew, R; Campbell, JB. 1981. Investigation of Environmental Factors and their relationship to populations of the stable fly, Stomoxys calcitrans (L.). Environ. Entomol. 10(5):798-800.

Rodríguez-Batista, Z; Leite, RC; Oliveira, PR; Lopes, CML; Borges, MLF. 2005. Populational dynamics of Stomoxys calcitrans (Linneaus) (Diptera: Muscidae) in three biocenosis, Minas Gerais, Brazil. Veterinary Parasitology. 130(3):343-346.

Salama, HS; Abde-Elgawad, MM. 2010. Spatial patterns of the red palm weevil and applied entomopathogenic nematode Heterorhabditis bacteriophora. Arch. Phytopathol. Plant Prot. 43(7):689-699. 
SENASA (Servicio Nacional de Salud Animal). 2012. Informe Estadístico denuncias por Mosca del Establo. Informe anual Dirección Regional de Salud Animal Región Huetar Norte. Costa Rica. MAG. 8 p.

Socías, MG; Liljesthröm, GG; Casmuz, AS; Murúa, MG; Gastaminza, G. 2014. Density and spatial distribution of different development stages of Sternechus subsignatus Boheman (Coleoptera: Curculionidae) in soybean crops. Crop Prot. 65: 15-20.

Stubbins, FL; Seiter, NJ; Greene, JK. 2014. Developing sampling plans for invasive Megacopta Cribraria (Hemiptera: Plataspidae) in soybeans. J. Econ. Entomol. 107(6):2213-2221.

Taylor, D; Moon, R; Darrel, M. 2012. Economic Impact of Stable Flies (Diptera: Muscidae) on Dairy and Beef Cattle Production. J. Med. Entomol. 49(1):198-209. DOI: http://dx.doi.org/10.1603/ ME10050.

Taylor, LR. 1984. Assesing and interpreting the spatial distributions of insect populations. Annu. Rev. Entomol. 29:321-359.

Taylor, DB; Berkebile, DR; Scholl, PJ. 2007. Stable fly population dynamics in Eastern Nebraska in relation to climatic variables. J. Med. Entomol. 44(5):765-771.

Taylor, DB; Moon, RD; Campbell, JB; Berkebile, DR; Scholl, PJ; Broce, JB; Hogsette, JA. 2010. Dispersal of Stable Flies (Diptera: Muscidae) From Larval Development Sites in a Nebraska Landscape. Environ. Entomol. 39(4):1101-1110.

Veccol, D; Pinedol, R; Fernández, M. 2015. Métodos de muestreo para Pseudophilothrips sp. (Tubulifera: Phlaeothripidae) y sus enemigos naturales: I. Muestreo secuencial enumerativo. Rev. Protec. Veg. 30(1):6-13.

Zhang, Y; Wang, H; Ran, K; Wang, S. 2015. Spatial pattern and sampling technique of distribution of eggs Psylla chinensis first generation. J. Sci. Fruit. 31(1):986-990.

Zheng- Yong, L; EnGuo, W; Jianjun, C; GuoJun, L; XiaoMing, Y. 2015. The spatial distribution pattern and sampling technique of Bemisia tabaci in leaf mustard fields. Agric. Zhejiangensis Act. 27(2):589-592.
Zumbado, MA. 2006. Dípteros de Costa Rica y la América Tropical. Instituto Nacional de Biodiversidad (INBio), Heredia, Costa Rica. 267 p.

Zumpt, F. 1973. The Stomoxyine Biting Flies of the World (Diptera: Muscidae). Taxonomy, biology, economic importance and control measures. Stuttgart Fischer, USA. 20(4-5):433. 